University of Nebraska - Lincoln

DigitalCommons@University of Nebraska - Lincoln

\title{
Spatial variations in local switching parameters of ferroelectric random access memory capacitors
}

D. $\mathrm{Wu}$

North Carolina State University

I. Kunishima

Toshiba Corporation

S. Roberts

University of Nebraska-Lincoln

Alexei Gruverman

University of Nebraska-Lincoln, agruverman2@unl.edu

Follow this and additional works at: https://digitalcommons.unl.edu/physicsgruverman

Part of the Physics Commons

Wu, D.; Kunishima, I.; Roberts, S.; and Gruverman, Alexei, "Spatial variations in local switching parameters of ferroelectric random access memory capacitors" (2009). Alexei Gruverman Publications. 53.

https://digitalcommons.unl.edu/physicsgruverman/53

This Article is brought to you for free and open access by the Research Papers in Physics and Astronomy at DigitalCommons@University of Nebraska - Lincoln. It has been accepted for inclusion in Alexei Gruverman Publications by an authorized administrator of DigitalCommons@University of Nebraska - Lincoln. 


\title{
Spatial variations in local switching parameters of ferroelectric random access memory capacitors
}

\author{
D. Wu, ${ }^{1}$ I. Kunishima, ${ }^{2}$ S. Roberts, ${ }^{3}$ and A. Gruverman ${ }^{3, a)}$ \\ ${ }^{1}$ North Carolina State University, Raleigh, North Carolina 27695, USA \\ ${ }^{2}$ Toshiba Corporation, Yokohama 235-8522, Japan \\ ${ }^{3}$ University of Nebraska-Lincoln, Lincoln, Nebraska 68588, USA
}

(Received 1 June 2009; accepted 9 July 2009; published online 1 September 2009)

\begin{abstract}
Spatially resolved studies of the switching behavior of micrometer scale $\mathrm{Pb}(\mathrm{Zr}, \mathrm{Ti}) \mathrm{O}_{3}$ capacitors have been performed by piezoresponse force microscopy (PFM). PFM spectroscopy and bias-dependent imaging of domain patterns have been used to investigate variability in local switching parameters and address the capacitor scaling effect on switching. It was found that average coercive voltage and imprint bias are independent of capacitor size and are similar to the corresponding parameters obtained by polarization hysteresis measurements. This can be attributed to the slow switching kinetics during quasistatic PFM measurements. The obtained results demonstrate a possibility of testing the submicron capacitors in real devices. () 2009 American Institute of Physics. [DOI: 10.1063/1.3192354]
\end{abstract}

Investigation of the switching behavior of micrometer scale capacitors is important both for fundamental understanding of the scaling effect in ferroelectrics and for development of high-density ferroelectric random access memory (FeRAM) technology. ${ }^{1}$ Ferroelectric polarization reversal is controlled by nucleation probability and domain wall velocity. However, with decrease in capacitor size, relative contribution of these mechanisms may vary leading to a change in the switching dynamics and switching speed. ${ }^{2}$ Macroscopic switching parameters, such as coercive bias and switching time obtained from polarization hysteresis and transient current measurements, represent a sum of elementary nucleation and wall movement events averaged over the capacitor volume. However, these methods are hardly applicable to the submicron capacitors due to the increased parasitic capacitance and significantly worse signal-to-noise ratio. This problem can be overcome by using in situ compensation of the parasitic signal in the scanning probe microscopy setup. ${ }^{3}$ However, as these methods provide no spatially resolved information on domain dynamics, they are of little help to improve the present understanding of the switching behavior of the finite size ferroelectric structures and to predict how the capacitor scaling affect its coercive voltage and remanent polarization (imperative for commercial FeRAM design).

Piezoresponse force microscopy (PFM) presents a unique possibility of nondestructive imaging of polarization distribution in ferroelectric capacitors with the nanoscale spatial resolution effectively emulating the switching conditions in real FeRAM devices. ${ }^{4}$ One of the most important issues related to the development of high-density FeRAM devices is the correlation between the global and local hysteresis loops and its relation to capacitor scaling. In other words, is it possible to predict how the average coercive bias, imprint, and remanent polarization will scale with capacitor size based on the local hysteresis loop measurements? In this paper, to address this problem, we apply PFM to visualize the domain evolution in microscale capacitors as a function of an applied electrical field and capacitor size. This method,

${ }^{\text {a)}}$ Electronic mail: alexei_gruverman@unl.edu. similar to the one developed by Hong et al., ${ }^{5}$ allows direct imaging of spatial distribution of local switching parameters providing information complementary to the local spectroscopic measurements. ${ }^{6}$ In addition, we perform image analysis of the PFM data to obtain the global PFM hysteresis loop in conjunction with two-dimensional mapping of the local switching parameters. From direct comparison of the polarization hysteresis and global PFM hysteresis data, it is shown that in spite of the spatial variations in local coercive voltages and qualitatively different switching dynamics the capacitor scaling does not lead to a significant change in the average switching parameters.

Polycrystalline $100-\mathrm{nm}$ thick $\mathrm{Pb}(\mathrm{Zr}, \mathrm{Ti}) \mathrm{O}_{3}$ (PZT) films were grown on a Ir bottom electrode with a thin $\mathrm{SrRuO}_{3}$ (SRO) layer by metal-organic chemical-vapor deposition. The PZT films were randomly oriented and had an average grain size of about $50 \mathrm{~nm}$. A sputter-deposited 30-nm thick $\mathrm{SRO} / \mathrm{IrO}_{2}$ stacked layer was used to fabricate top electrodes with the lateral dimensionsin in the range from $2 \times 2 \mu \mathrm{m}^{2}$ to $0.3 \times 0.3 \mu \mathrm{m}^{2}$.

The experimental setup is based on a commercial atomic force microscope (Asylum Research MFP-3D), equipped with Keithley 237 function generator and Stanford Research Systems lock-in amplifier. The dc and ac bias signals have been applied to the PZT capacitors via a Pt-coated Si cantilever (NSC14/Pt, MikroMasch). The same cantilevers have been used for PFM domain imaging in the capacitors as small as $0.3 \times 0.3 \mu \mathrm{m}^{2}$ (Fig. 1). The bias-dependent domain switching behavior has been studied by superimposing a high-frequency imaging ac bias $\left(0.3 \mathrm{~V}_{\mathrm{rms}}, 20 \mathrm{kHz}\right)$ and a low-frequency $(10 \mathrm{mHz})$ switching bias using a pulse train shown in Fig. 1. A set of PFM images obtained at an incrementally changing external field has been used to obtain information on field-dependent evolution of domain structure during polarization reversal and to address the capacitor size effect on domain dynamics.

Structural imperfections associated with polycrystalline nature of the PZT capacitors result in spatial variations of the local switching parameters at the nanoscale level. Local hysteresis loops obtained in three different locations and shown 

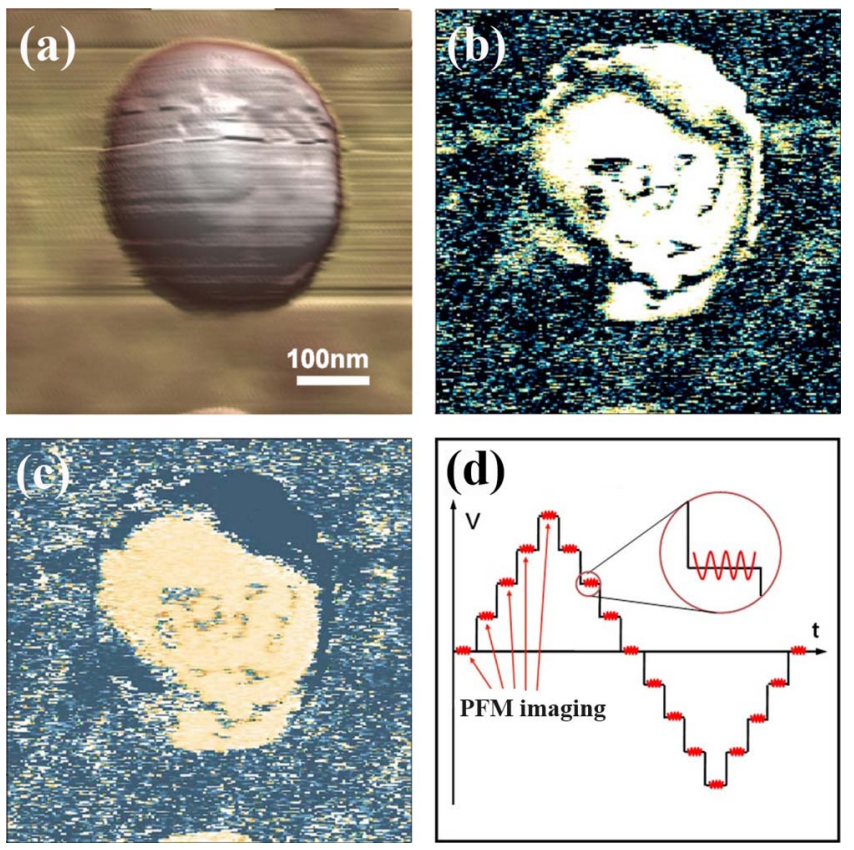

FIG. 1. (Color online) (a) Topographic, (b) PFM amplitude, and (c) PFM phase images of the $0.33 \times 0.33 \mu \mathrm{m}^{2}$ PZT capacitor. (d) Voltage pulse train used for BD-PFM studies.

in Fig. 2(a) illustrate this point. All three locations are characterized by different switching parameters, such as imprint and coercive bias, which are quite different from the parameters of the typical polarization $P-V$ hysteresis loops measured by the Sawyer-Tower method [Fig. 2(b)]. The switching spectroscopy PFM (SS-PFM) approach ${ }^{7}$ provides further insight into the spatial variability of switching. A twodimensional map of local imprint bias [Fig. 2(c)] has been generated by acquiring local hysteresis loops [similar to those in Fig. 2(a)] at each point while rastering the top electrode surface with subsequent analysis. Spatial distribution
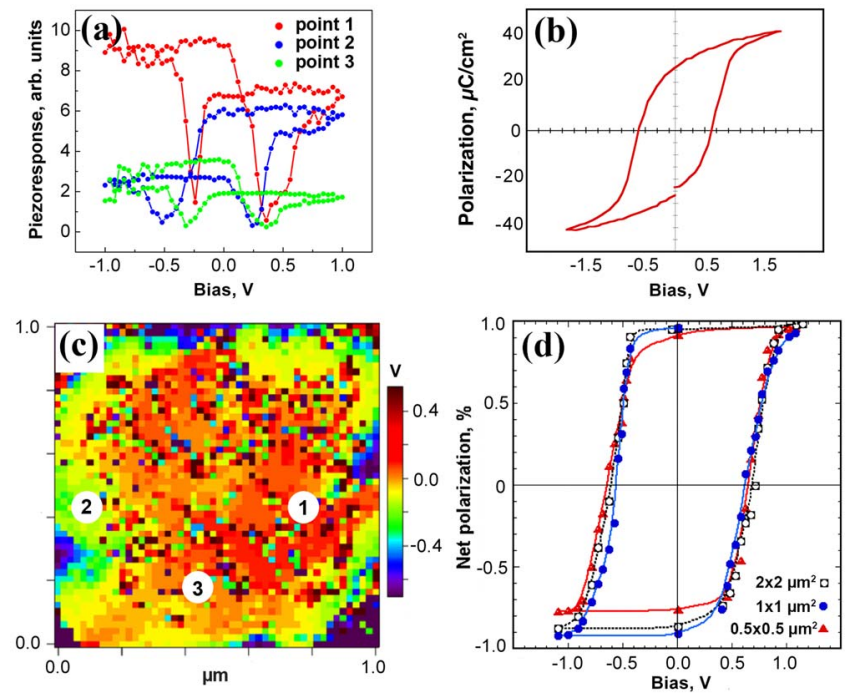

FIG. 2. (Color online) (a) Local PFM hysteresis loops measured in different locations on the surface of the $1.0 \times 1.0 \mu \mathrm{m}^{2}$ PZT capacitor. Loop numbers correspond to location numbers shown in (c). (b) Conventional $P-E$ hysteresis loop measured in the same type of a PZT capacitor but larger dimensions $\left(65 \times 65 \mu \mathrm{m}^{2}\right)$. (c) $2 \mathrm{D}$ map of imprint bias in the $1.0 \times 1.0 \mu^{2} \mathrm{PZT}$ capacitor generated by SS-PFM. (d). Global PFM hysteresis loops measured in capacitors of different dimensions.

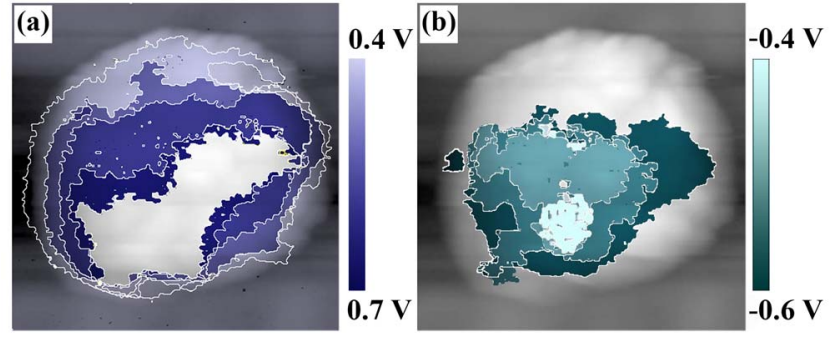

FIG. 3. (Color online) Domain structure evolution delineated by means of BD-PFM in the $0.5 \times 05 \mu \mathrm{m}^{2}$ capacitor (scan size is $0.65 \times 0.65 \mu \mathrm{m}^{2}$ ). (a) Switching to a positive polarization state. Domain profiles are shown for $\mathrm{dc}$ bias of $0.40,0.55,0.60$, and $0.70 \mathrm{~V}$. (b) Switching to a negative polarization state. Domain profiles are shown for dc bias of $-0.40,-0.45,-0.50$, and $-0.60 \mathrm{~V}$.

of the imprint and coercive bias gives a sense of the expected domain dynamics during switching.

To clarify the relationship between macroscopic polarization hysteresis and local switching parameters, we employ a bias-dependent PFM (BD-PFM) approach to visualize domain structure evolution as a function of the applied voltage and capacitor size. Figures 3 and 4 show profiles of domain patterns developing during switching in $0.5 \times 0.5$ and $2 \times 2 \mu \mathrm{m}^{2}$ PZT capacitors, respectively, under an external bias incrementally increasing in the positive and negative directions. It can be seen that larger capacitors exhibit a number of nucleation sites. For smaller capacitors, the switching is mainly dominated by domain wall motion. An interesting feature here is asymmetry of switching in the positive and negative directions. While for negative direction switching proceeds via lateral expansion of a singe domain nucleating in the capacitor center, for the positive direction it starts from the electrode edge via growth of residual domains. Similar effect of the electrode edge, although to a much smaller extent, can be observed in the larger capacitors. This observation is consistent with the imprint behavior in the electrode edge region detected by local hysteresis loop measurements. This effect can be explained by a higher defect concentration at the perimeter region generated during top electrode fabrication. ${ }^{8}$ Another feature worthwhile mentioning is roughening of domain wall topology appearing as a number of nanoscale protrusions with a curvature radius of $10-30 \mathrm{~nm}$. This observation implies that the domain walls do not propagate in a continuous manner but rather by triggering switching in nanoscale regions in front of the wall. As the average grain size in the PZT layer is $\sim 50 \mathrm{~nm}$, it can be also concluded that these nanoscale switched regions corre-
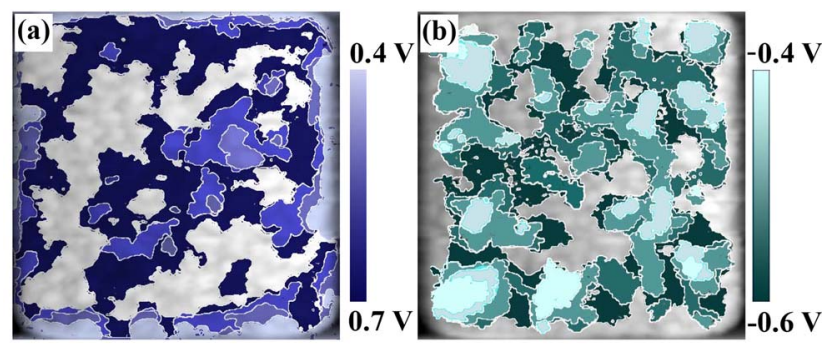

FIG. 4. (Color online) Domain structure evolution delineated by means of BD-PFM in the $2.0 \times 2.0 \mu \mathrm{m}^{2}$ capacitor. (a) Switching to a positive polarization state. Domain profiles are shown for dc bias of 0.4, 0.5, 0.55, and 0.7 V. (b) Switching to a negative polarization state. Domain profiles are shown for dc bias of $-0.45,-0.50,-0.55,-0.60$, and $-0.65 \mathrm{~V}$. 
spond to individual grains. This effect can be attributed to a short-range electrostatic or mechanical coupling between the adjacent grains reported elsewhere. ${ }^{9,10}$ Given that the coercive voltage of a grain depends upon its crystallographic orientation, ${ }^{11}$ this type of domain wall propagation is consistent with the nucleation-limited switching mechanism reported in polycrystalline PZT capacitors, which infers a wide distribution of local switching parameters. ${ }^{12,13}$

We made a step further to bridging the macroscopic and local switching behavior by numerically analyzing the BDPFM imaging data in Figs. 3 and 4. Specifically, using commercial software (WSXM v2.2, Nanotec Electronica), the PFM phase and amplitude images have been converted to the switched capacitor volume for a given value of the applied bias. Using this method we obtained global PFM hysteresis loops [Fig. 2(d)], which are analogous to the conventional polarization $P-V$ hysteresis loop. What is important is that the global PFM loops can be measured for capacitors with dimensions well into the submicron range thus the overcoming the limitations of the $P-E$ measurements and allowing us to address the capacitor scaling effect on the global switching parameters. Figure 2(d) shows the global PFM hysteresis loops for capacitors of three different dimensions. Surprisingly, hysteresis loops width, coercive voltages and imprint are very similar for all three capacitor sizes. These values are also close to the parameters of the $P-V$ loops measured in capacitors of much larger dimensions [Fig. 2(b)]. The capacitor scaling effect is manifested only in the decrease of the remanent negative polarization for $0.5 \times 0.5 \mu \mathrm{m}^{2}$ capacitors (by about 20\%) due to the pinned positive polarization along the capacitor perimeter. Indeed, local hysteresis loop measurements [Fig. 2(a)], SS-PFM mapping [Fig. 2(c)] and BD-PFM imaging data (Fig. 3) all indicate that the perimeter area exhibits positive imprint, which precludes its complete switching.

The absence of size dependent hysteresis behavior is in striking contrast to the strong scaling effect on the switching time. ${ }^{2}$ One might expect that a difference in switching speed would be also reflected in the size dependence of the coercive voltage. As hysteresis loop measurements have been done in a quasi-static regime, this discrepancy is a strong indication that the scaling effect is determined by the switching kinetics. In the quasistatic hysteresis loop measurements, switching is typically initiated in a very few sites with significantly reduced activation energy. The density of these sites is rather low [from our PFM studies it can be estimated as $\left.(2-5) \times 10^{12} \mathrm{~m}^{-2}\right]$. In this case, switching mainly proceeds via lateral domain growth driven by nanoscale domain formation in front of the moving domain walls with characteristic spacing of the order of the grain size, i.e., $\sim 50 \mathrm{~nm}$. This mechanism would not reveal any scaling effect in the capacitors of $0.5 \times 0.5 \mu \mathrm{m}^{2}$ and larger. However, in the pulsed measurements of the switching times, a competition between two rate-limiting parameters-wall speed and nucleation rate-will result in significant scaling effect as has been verified by experimental PFM studies. ${ }^{2}$
In summary, the switching behavior of micrometer scale PZT capacitors has been studied by employing local spectroscopic PFM measurements in conjunction with BD-PFM imaging. The applied approach allowed addressing the capacitor scaling behavior and bridging the global switching parameters and spatially resolved features of domain switching dynamics. It has been found that average coercive voltage and imprint bias extracted from global PFM hysteresis loops are independent of capacitor size and are similar to the corresponding parameters obtained by polarization hysteresis measurements. This finding, which is in stark contrast to the profound capacitor size effect on the switching rate, is attributed to the kinetic nature of the scaling effect. If the switching proceeds mainly via lateral domain growth, local variations in the threshold voltages at the length scales much smaller than the capacitor size do not result in the sizedependent average coercive voltage and imprint. On the other hand, these variations will lead to significant capacitor scaling effect in the case of a competition between walllimited and nucleation-limited switching mechanisms. It has been shown that decrease in the remanent polarization in smaller capacitors is a result of unswitchable polarization along the perimeter area likely due to the processing damage introduced into the PZT layer. This detrimental effect, which is expected to worsen with further capacitor size reduction, needs to be taken into account while developing high-density FeRAM devices.

This work was supported by the National Science Foundation (Grant No. NIRT DMR-0403871), the Nebraska Center for Materials and Nanoscience at University of NebraskaLincoln and Toshiba Corporation. The authors express their gratitude to R. Proksch and J. Bemis, Asylum Research, for their help in generating the SS-PFM map, to A. I. Kingon for useful discussions, and to J. F. Scott for valuable comments.

${ }^{1}$ J. F. Scott, Ferroelectric Memories (Springer, Berlin, 2000).

${ }^{2}$ A. Gruverman, D. Wu, and J. F. Scott, Phys. Rev. Lett. 100, 097601 (2008).

${ }^{3}$ S. Tiedke, T. Schmitz, K. Prume, A. Roelofs, T. Schneller, U. Kall, R. Waser, C. S. Ganpule, V. Nagarajan, A. Stanishevsky, and R. Ramesh, Appl. Phys. Lett. 79, 3678 (2001).

${ }^{4}$ D. J. Kim, J. Y. Jo, T. H. Kim, S. M. Yang, B. Chen, Y. S. Kim, and T. W. Noh, Appl. Phys. Lett. 91, 132903 (2007).

${ }^{5}$ S. Hong, E. L. Colla, E. Kim, D. V. Taylor, A. K. Tagantsev, P. Muralt, K. No, and N. Setter, J. Appl. Phys. 86, 607 (1999).

${ }^{6}$ S. Jesse, A. P. Baddorf, and S. V. Kalinin, Appl. Phys. Lett. 88, 062908 (2006).

${ }^{7}$ P. Bintachitt, S. Trolier-McKinstry, K. Seal, S. Jesse, and S. V. Kalinin, Appl. Phys. Lett. 94, 042906 (2009).

${ }^{8}$ M. Grossmann, O. Lohse, D. Bolte, R. Waser, W. Hartner, G. Schindler, C. Dehm, N. Nagel, J. Joshi, N. Solayappan, and G. Derbenwick, Integr. Ferroelectr. 22, 95 (1998).

${ }^{9}$ I. Stolichnov, L. Malin, E. Colla, A. K. Tagantsev, and N. Setter, Appl. Phys. Lett. 86, 012902 (2005).

${ }^{10}$ B. J. Rodriguez, A. Gruverman, A. I. Kingon, R. J. Nemanich, and J. S. Cross, Appl. Phys. A: Mater. Sci. Process. 80, 99 (2005).

${ }^{11}$ S. V. Kalinin, A. Gruverman, and D. A. Bonnell, Appl. Phys. Lett. 85, 795 (2004).

${ }^{12}$ X. F. Du and I. W. Chen, Appl. Phys. Lett. 72, 1923 (1998).

${ }^{13}$ A. Tagantsev, I. Stolichnov, N. Setter, J. S. Cross, and M. Tsukada, Phys. Rev. B 66, 214109 (2002). 